\title{
O novo modelo Front Velocity aplicado na modelagem da separação de enantiômeros via processo Leito Móvel Simulado
}

\author{
Anderson L. J. Bihain \\ Universidade Federal do Pampa, Campus Bagé, UNIPAMPA \\ 96413-170, Bagé, RS \\ E-mail: andersonbihain@unipampa.edu.br
}

\author{
Leôncio D. T. Câmara Antônio J. da Silva Neto \\ Instituto Politécnico da Universidade Estadual do Rio de Janeiro - IPRJ \\ 28625-570, Nova Friburgo, RJ \\ E-mail:dcamara@iprj.uerj.br_ajsneto@iprj.uerj.br
}

\begin{abstract}
Resumo: Devido as propriedades terapêuticas dos enantiômeros de um fármaco racêmico, surge a necessidade de separá-los. Entre os processos utilizados para a separação desse tipo de composto, o Leito Móvel Simulado (LMS) tem recebido destaque, por ser um processo contínuo e contracorrente que apresenta um grande eficiência de separação. Os fármacos racêmicos em geral são caros e por isso existe o interesse em evitar possíveis desperdícios ocasionados por uma operação inadequada do equipamento utilizado na separação. Para determinar os parâmetros de operação do LMS é necessário predizer o transporte de massa que ocorre no interior das colunas cromatográficas que compõe o LMS. Atualmente os modelos usados na predição do transporte de massa nesse tipo de processo são compostos por sistemas de equações diferenciais parciais e apresentam um alto custo computacional, além de que necessitam dado obtidos previamente através da realização de experimentos de equilíbrio que são interpretados por isotermas de adsorção. Neste trabalho, para modelar processo de separação em LMS a nova abordagem Front Velocity foi proposta. Esta é composta por equações diferenciais ordinárias e não se utiliza de isotermas de adsorção e consequentemente não se faz necessário a realização de experimentos de equilíbrio. Na cromatografia preparativa um experimento de pulso é conduzido, e do cromatograma resultante sã determinadas via aplicação do problema inverso os parâmetros globais de transferência de massa do modelo Front Velocity.. Na segunda etapa os parâmetros obtidos na caracterização da coluna cromatográfica foram utilizados na simulação do processo LMS, obtendo os perfis de separação dos compostos da mistura estudada. Para a validação da modelagem empregada, os resultados simulados foram comparados com dados experimentais da separação dos enantiômeros do racemato Cetamina (Santos, et al., 2004) e também confrontados com as simulações obtidas a partir de modelos clássicos. Os resultados obtidos mostraram que o modelo Front Velocity possui uma concordância razoável com os dados experimentais em particular quando foi utilizada a cinética de Langmuir como mecanismo de transferência de massa. Da mesma forma mostrou perfis de separação semelhantes aos obtidos pela modelagem clássica usando apenas equações diferenciais ordinárias e exigindo um tempo de simulação computacional aproximadamente vinte vezes menor.
\end{abstract}

Palavras chave: Modelagem e Simulação, Leito Móvel Simulado, Problema inverso.

\section{INTRODUÇÃO}

Entre as variadas técnicas de separação existentes está o Leito Móvel Simulado, criado na década de sessenta pela Universal Oil Products (EUA Patente N ${ }^{\circ}$ US002985589, 1961). Esta recebe destaque por ser uma poderosa ferramenta capaz de separar misturas de difícil separação, quando a diferença de afinidade entre as moléculas é muito pequena, e também pela possibilidade de ser operado continuamente exigindo menos solvente do que a cromatografia em batelada (Gal, et al., 2005). O número de estudos ao redor do mundo e a quantidade de publicações têm consagrado e evidenciado o potencial dessa ferramenta cromatográfica.

Para operar o LMS é necessário um estudo aprofundado, dado que é necessário determinar algumas condições operacionais, tais como a taxa de fluxo em cada seção, a 
concentração da alimentação e o intervalo de troca da posição das correntes de fluxo (Gonçalves, 2008). Para resolver essa questão muitos autores formularam modelos capazes de predizer o processo de separação em LMS com erros estatisticamente aceitáveis em relação a dados experimentais. Em geral os modelos utilizados pelos pesquisadores são robustos e eficientes, porém possuem um alto custo computacional associado.

Para predizer os perfis de separação em um intervalo de tempo menor dos compostos de uma mistura submetida ao processo de separação cromatográfica por adsorção em LMS, a nova abordagem Front Velocity foi proposta. Esta não necessita de experimentos de equilíbrio e aplicação de isotermas para caracterizar os componentes envolvidos no processo, além de possibilitar uma simulação rápida do processo.

Para a validação da modelagem proposta, os resultados foram comparados com os dados experimentais obtidos por Santos (2004) e Santos et al. (2004) e com as simulações obtidas pela modelagem clássica realizada pelos mesmos autores.

\section{MODELAGEM}

Santos (2004) e Santos et al. (2004) usaram um LMS para a separação dos enantiômeros do racêmico Cetamina, composto de oito colunas cromatográficas interligadas em série, distribuídas duas a duas por zona, sendo que cada coluna tem $0,77 \mathrm{~cm}$ de diâmetro e $20 \mathrm{~cm}$ de comprimento como está representado na Figura 1. O enantiômero mais adsorvido (R) é coletado no extrato (Ex), enquanto o enantiômero menos adsorvido (S) é coletado no rafinado (R).

a)

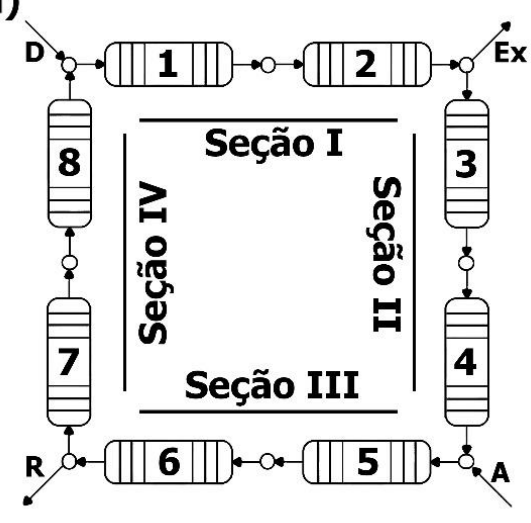

b)

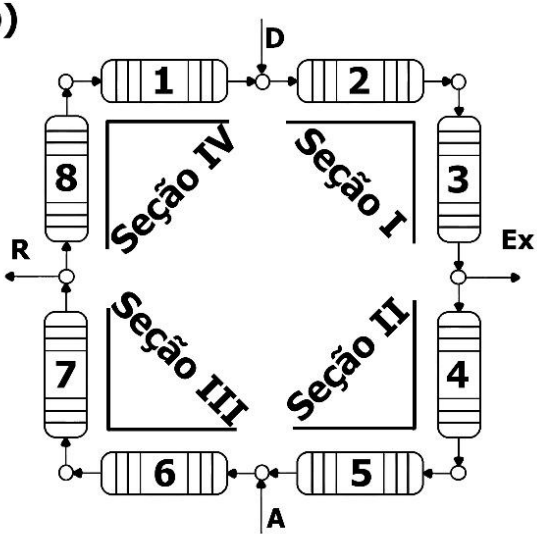

Figura 1:Esquema de uma unidade de LMS com distribuição 2-2-2-2.

Nas fases estacionária e móvel foram utilizados respectivamente, MCTA (Acetato de celulose microcristalino) e etanol. Santos et al. (2004) também usaram um sistema de análise CLAE (Cromatografia Líquida de Alta Eficiência), onde injetaram amostras de Cetamina para calibração e determinação da pureza, além de realizarem experimentos de equilíbrio de adsorção para a caracterização das colunas cromatográficas com auxílio de uma isoterma linear. A coluna do HPLC utilizada possui $0,46 \mathrm{~cm}$ de diâmetro e $20 \mathrm{~cm}$ de comprimento.

Para representar a transferência de massa que ocorre no interior de uma coluna cromatográfica durante o processo de separação, a nova abordagem Front Velocity foi desenvolvida. Esta estabelece que a convecção é a fase dominante no transporte de soluto ao longo da coluna cromatográfica. O "Front Velocity" é um modelo discreto (etapas) em que a vazão determina o avanço da fase líquida ao longo da coluna. Para determinar a velocidade com que a fase líquida percola a coluna porosa $(v)$, a relação obtida através do fluxo volumétrico da fase móvel através do meio poroso descrita pela Eq. 1 é utilizada,

$$
v=\frac{Q}{\varepsilon A_{T}}
$$

onde, $Q$ é a vazão volumétrica, $\varepsilon$ é a porosidade e $A_{T}$ é a área total da coluna porosa (Dados estes que são obtidos experimentalmente). 
Para realizar o cálculo da transferência de massa, a coluna cromatográfica foi discretizada em volumes de controle de comprimento $\xi$ que se movem ao longo da coluna com a mesma velocidade do eluente, conforme pode ser observado na figura 2.

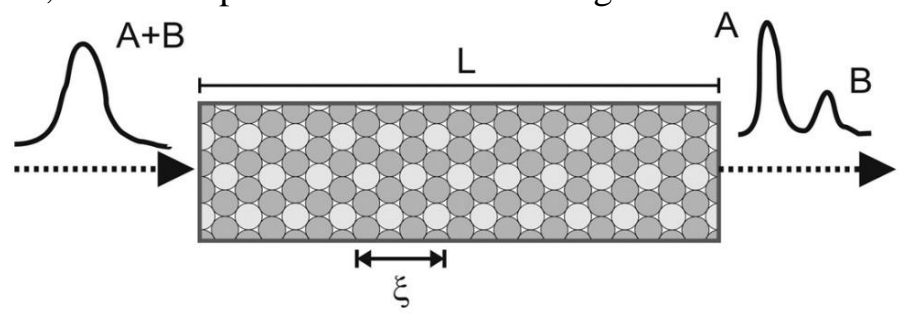

Figura 2: Coluna cromatográfica de comprimento L e volume de controle de comprimento $\xi$.

O tempo necessário $(\Delta t)$ para a fase líquida se mover ao longo de cada volume de controle é obtido a partir da Eq. 2 onde $V$ é o volume total da coluna e $n$ é o número de volumes de controle.

$$
\Delta t=\frac{\varepsilon \cdot V}{n \cdot Q}
$$

Para realizar o cálculo da transferência de massa foi assumido o equilíbrio imediato nos volumes de controle, de forma que os efeitos da dispersão axial e da resistência à transferência de massa foram desconsiderados. Para caracterizar a cinética de transferência de massa do soluto entre a fase líquida e a fase sólida foi assumido o modelo global de cinética de transferência de massa (Eq. 3).

$$
\left(\frac{d C}{d t}\right)_{\xi}=-r_{q} \quad \text { e } \quad\left(\frac{d q}{d t}\right)_{\xi}=r_{q}
$$

Para descrever o comportamento dos sítios de adsorção das colunas cromatográficas, dois diferentes mecanismos cinéticos foram adotados. No primeiro (Eq. 4) foi assumida uma relação linear, onde não existe uma capacidade máxima de adsorção $Q_{m}$, enquanto que no segundo caso uma equação cinética (Eq. 5) que contempla a saturação das colunas foi utilizada.

$$
\begin{aligned}
& r_{q}=k_{\text {ads }} \cdot C-k_{d e s} \cdot q \\
& r_{q}=k_{\text {ads }} \cdot C(q m-q)-k_{d e s} \cdot q
\end{aligned}
$$

Nas equações acima os termos $C, q, q_{m}, k_{a d s}$ e $k_{d e s}$ são respectivamente, a concentração na fase líquida e sólida, a capacidade máxima de adsorção da fase sólida e a constante cinética global de transferência de massa de adsorção e de dessorção.

Para determinar os parâmetros $k_{a d s}$ e $k_{\text {des }}$ o cromatograma resultante da análise do experimento de pulso em CLAE realizada por Santos(2004) foi utilizado. Estes dados foram combinados com as Eqs. 3, 4 e 5 e com a ferramenta inversa, Random Restricted Window (R2W) (Câmara \& Silva Neto, 2008).

O R2W é considerado um método inverso estocástico simples, o qual utiliza um algoritmo de busca com uma distribuição aleatória. Após a obtenção dos parâmetros cinéticos de transferência de massa, o próximo passo foi realizar a simulação da separação dos enantiômeros no equipamento LMS.

Na segunda etapa o modelo das Eqs. 3, 4 e 5 foi aplicado para cada uma das equações colunas do LMS para descrever o transporte de massa. Além disso, devido as diferentes vazões em cada seção e as entradas e retiradas de material, faz-se necessário realizar o cálculo das vazões em cada uma das seções do LMS e o balanço de massa antes do início do processo e a cada troca de posição das correntes. Para realizar tal tarefa as considerações a baixo se fazem necessárias.

Como no LMS temos duas correntes de entrada e duas de saída, o balanço global de fluxo é necessariamente escrito da maneira a seguir.

$$
Q^{A}+Q^{D}=Q^{R}+Q^{E}
$$

Desta forma escrevemos as relações para calcular as vazões em cada seção e o balanço de massa para primeira coluna de cada seção.

Seção I:

$$
Q^{I}=Q^{I V}+Q^{D}, \quad C_{j E}^{I} \cdot Q^{I}=C_{j S}^{I V} \cdot Q^{I V}
$$


Seção II

$Q^{I I}=Q^{I}-Q^{E x}, \quad C_{j E}^{I I}=C_{j S}^{I}=C_{j}^{E x}$

Seção III

$Q^{I I I}=Q^{I I}+Q^{A}, \quad C_{j E}^{I I I} \cdot Q^{I I I I}=C_{j}^{A} \cdot Q^{A}+C_{j S}^{I I} \cdot Q^{I I}$

Seção IV

$Q^{I V}=Q^{I I I}-Q^{R}$,

$C_{j E}^{I V}=C_{j S}^{I I I}=C_{j}^{R}$

onde, $D, E x, A$ e $R$ correspondem respectivamente as correntes do dessorvente, extrato, alimentação e rafinado. E é a concentração de entrada (concentração na entrada da primeira coluna da seção), S é a concentração na saída da última coluna da seção e j é relativo ao composto da mistura (Ex: no caso de uma mistura racêmica os compostos são R e $S$ ).

\section{RESULTADOS E DISCUSSÕES}

Para validar o Front Velocity, os tempos de residência (experimentais) obtidos por Santos et al. (2004) no experimento de pulso foram utilizados para a otimização dos parâmetros cinéticos que caracterizam a coluna do LMS usados por esses pesquisadores. Uma vez caracterizada a coluna, os próprios modelos cinéticos foram aplicados a cada coluna do LMS para simular a separação enantiomérica da mistura, permitindo que os resultados fossem comparados com os obtidos por Santos et al. (2004) e Santos (2004).

As equações usadas na modelagem do LMS neste trabalho, foram resolvidas numericamente pelo método de Runge-Kutta de $4^{\circ}$ ordem e implementado em linguagem Fortran 90. O tempo total de simulação do LMS foi de aproximadamente 1,5 minutos em um computador com processador Intel Core i5 $(2.3 \mathrm{GHz})$ em uma malha de cálculo $d t=10^{-5}$, onde o número de divisões das colunas girou entorno de 300 a 1000 estágios de equilíbrio conforme apresentado nas tabelas Tabela 3.

Já as equações diferenciais parciais do modelo de equilíbrio dispersivo usado por Santos 2004, foram resolvidas através da sub-rotina publica PDECOL (Madsen \& Sincovec, 1979), que implementa o método de elementos finitos para discretização espacial e as equações diferenciais ordinárias foram resolvidas pelo integrador temporal GEARIB (Hindmarsh, 1976). O tempo total de simulação foi de aproximadamente 4 horas, utilizando um processador Intel Pentium IV (2.4GHz) em uma malha de cálculo de $d t=10^{-5}$ com cada coluna tendo sido dividida em 30 elementos.

Tabela 1:Parâmetros de transferência de massa obtidos a partir da aplicação da rotina inversa R2W ao modelo Front Velocity.

\begin{tabular}{ccccc}
\hline & \multicolumn{2}{c}{ Cinética Linear } & \multicolumn{2}{c}{ Cinética de Langmuir } \\
\hline & Enantiômero S & Enantiômero R & Enantiômero S & Enantiômero R \\
$\boldsymbol{k}_{\boldsymbol{1}}$ & 4,2061 & 4,61438 & 0,00218857 & 0,00247352 \\
$\boldsymbol{k}_{\mathbf{2}}$ & 3,4980 & 1,69159 & 0,05430671 & 0,02700426 \\
$\boldsymbol{K}_{e q}=\boldsymbol{k}_{\boldsymbol{l}} / \boldsymbol{k}_{\boldsymbol{2}}$ & 1,2024 & 2,72783 & 0,04030017 & 0,09159742 \\
$\mathbf{N}^{\circ}$ estágios & 505 & 505 & 505 & 505 \\
$\boldsymbol{q}_{\boldsymbol{m}}$ & - & - & 29,567 & 29,567 \\
$\mathbf{R}^{*}$ & $1,65 \mathrm{E}-06$ & $2,21 \mathrm{E}-05$ & $2,54 \mathrm{E}-05$ & $1,87 \mathrm{E}-06$ \\
\hline * é o resíduo quadrado entre a simulação e os dados experimentais
\end{tabular}

* é o resíduo quadrado entre a simulação e os dados experimentais

Na Tabela 1 estão representados os dados obtidos para os parâmetros de transferência de massa na solução do problema inverso para os dados do experimento de pulso na etapa de caracterização da coluna cromatográfica, enquanto nas Fig. 3 estão os resultados gráficos para o ajuste com o modelo Front Velocity quando adotado a cinética do tipo Langmuir (5).

Nas Fig. 3, fica notável a boa correlação entre os dados do experimento de pulso com a simulação feita pela abordagem Front Velocity para a cinéticas de e Langmuir, entretanto pelos resultados da tabela 1 é confirmado que entre os dois mecanismos cinéticos o de Langmuir obteve um melhor ajuste, pois este assume perfis diferentes de uma distribuição Gaussiana, já que os perfis de concentração experimental dos enantiômeros formam picos que apenas aproximam-se de uma distribuição gaussiana e não são distribuições perfeitamente Gaussianas. 


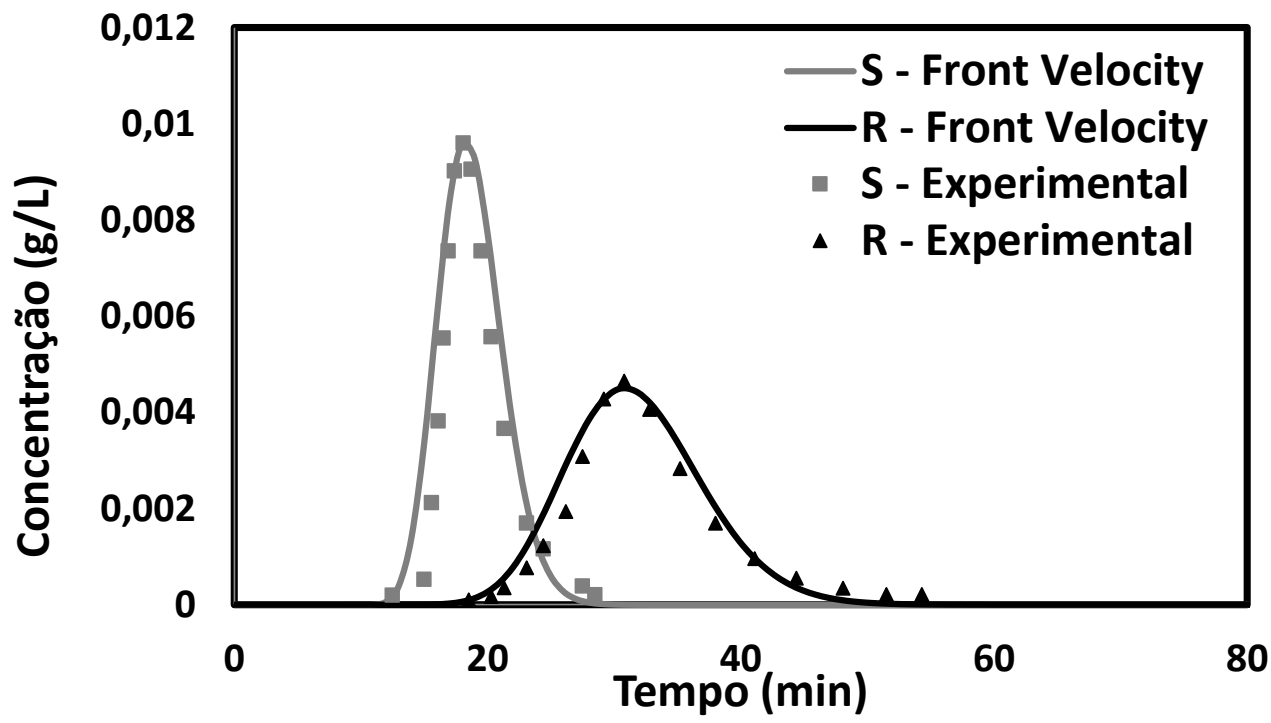

Figura 3:Cromatograma de injeção da cetamina racêmica na coluna de análise empacotada com MCTA; fase móvel etanol anidro, vazão $0,25 \mathrm{~mL} / \mathrm{min}$, "loop" de $20 \mu \mathrm{L}$, concentração da solução $1,5 \mathrm{~g} / \mathrm{L}$ em etanol anidro. Resultados simulados com Front Velocity com cinética de Langmuir, comparados aos dados experimentais.

Tabela 2:Condições experimentais usadas por Santos (2004) e Santos et al. (2004)

\begin{tabular}{cccccccccc}
\hline $\begin{array}{c}\text { Concentração da } \\
\text { alimentação }\end{array}$ & $\begin{array}{c}\text { Tempo de } \\
\text { troca em }\end{array}$ & \multicolumn{3}{c}{$\begin{array}{c}\text { Vazões das correntes } \\
(\mathbf{m L} / \mathbf{m i n})\end{array}$} & \multicolumn{4}{c}{$\begin{array}{c}\text { Vazões nas seções do LMS } \\
\text { (mL/min) }\end{array}$} \\
\cline { 3 - 11 }$(\mathbf{m g} / \mathbf{m L})$ & $(\mathbf{m i n})$ & $\boldsymbol{Q}^{\boldsymbol{E}}$ & $\boldsymbol{Q}^{\boldsymbol{R}}$ & $\boldsymbol{Q}^{\boldsymbol{A}}$ & $\boldsymbol{Q}^{\boldsymbol{D}}$ & $\boldsymbol{Q}_{\mathbf{1}}$ & $\boldsymbol{Q}_{\mathbf{2}}$ & $\boldsymbol{Q}_{\mathbf{3}}$ & $\boldsymbol{Q}_{\mathbf{4}}$ \\
\hline $\mathbf{1 , 5}$ & 25 & 0.47 & 0.43 & 0.18 & 1.10 & 1,10 & 0,63 & 0,81 & 0,38
\end{tabular}

Na tabela 2 estão dispostas as condições operacionais do LMS usadas por Santos (2004) e Santos et al. (2004) para a separação dos enantiômeros do anestésico Cetamina, enquanto que na Tabela 2 está à disposição o número de estágios de equilíbrio para as colunas em cada uma das quatro seções do LMS calculadas com o modelo Front Velocity com base nas vazões da tabela 1.

De posse dos parâmetros obtidos via aplicação do problema inverso (Tabela 1) e das condições operacionais (Tabela 2) o processo de separação em LMS foi simulado e comparado com os dados de Santos (2004), como pode ser observado nas Figs. 4, 5 e 6.

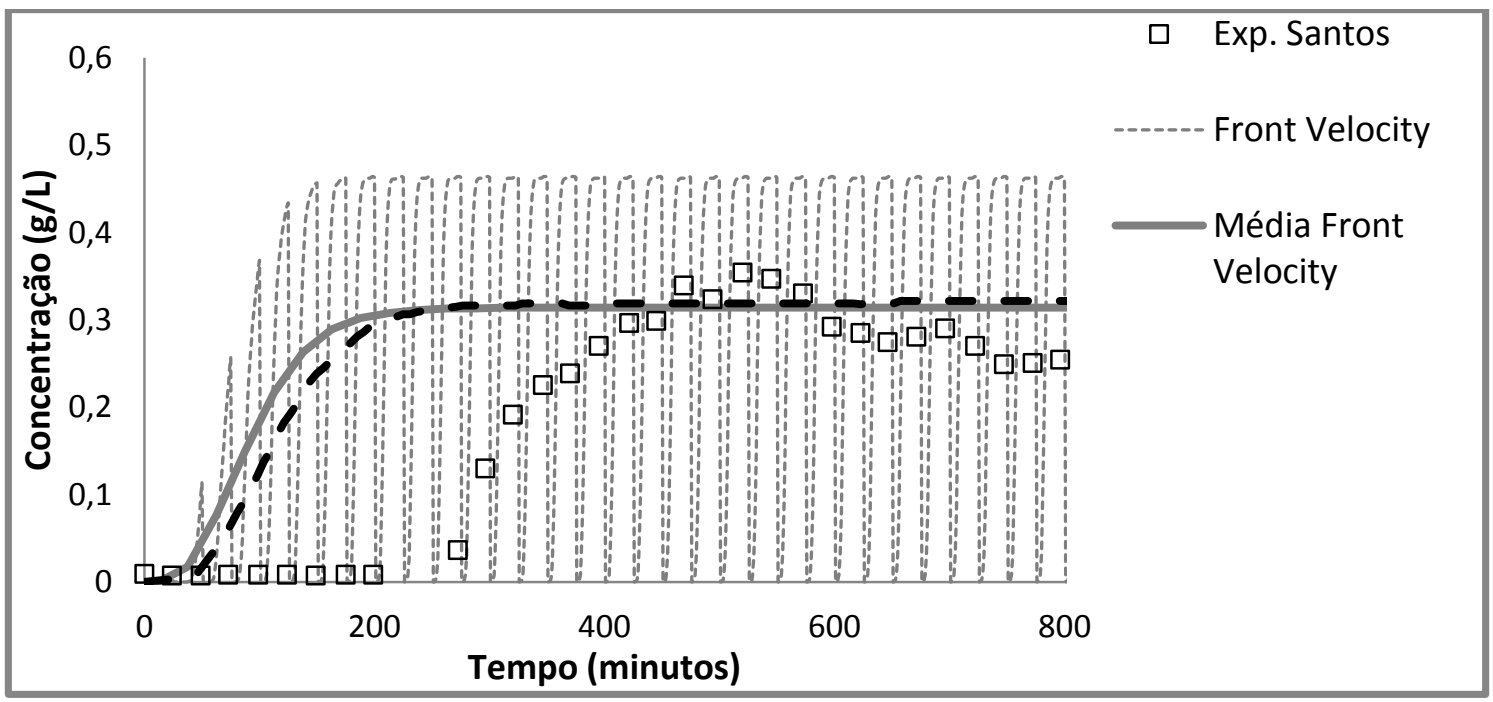

Figura 4:Evolução da concentração do enantiômero $\mathrm{S}$ na corrente do rafinado ao longo do tempo (transiente). 
A boa correlação entre resultados simulados com o modelo Front Velocity e o modelo clássico empregado por Santos et al e Santos (2004) durante todo o processo de separação podem ser observado nas Figuras 4 e 5. Também uma comparação com os dados experimentais das correntes de extrato e rafinado pode ser visualizada, onde observa-se uma discrepância entre os dados simulados e experimentais enquanto o processo está ainda no transiente e um melhor ajuste para quando atinge o estado pseudo estacionário.

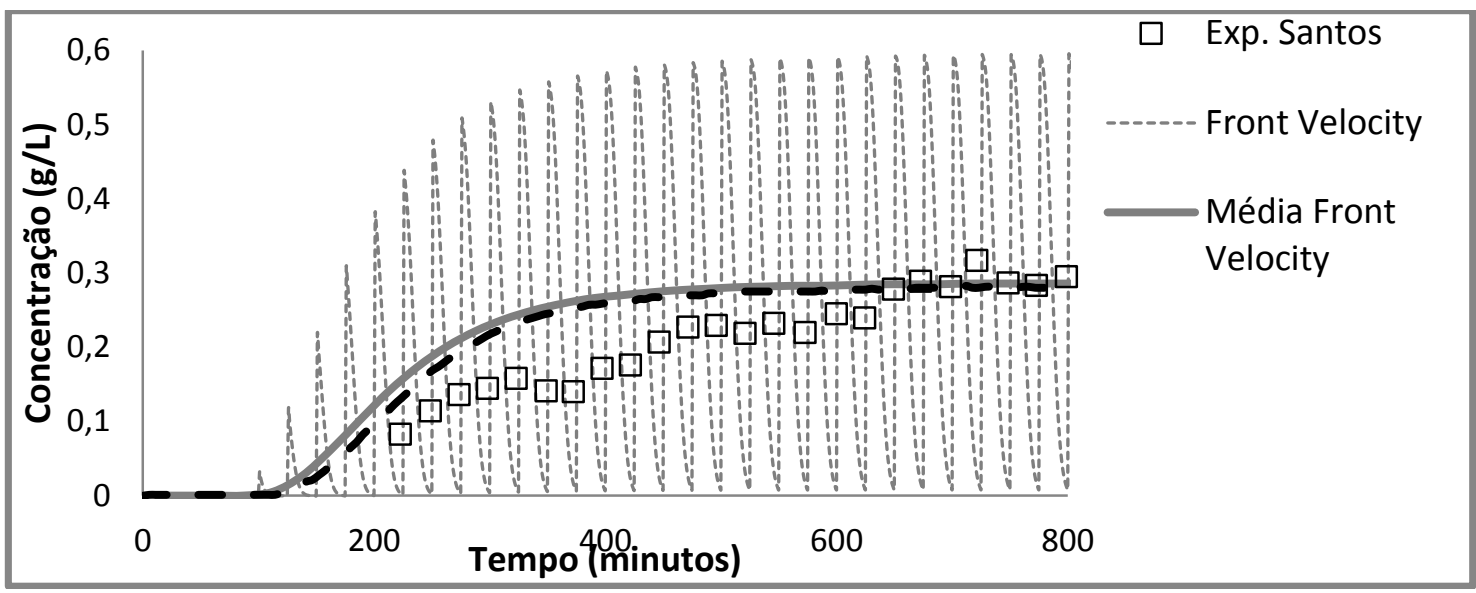

Figura 5:Evolução da concentração do enantiômero R na corrente do extrato ao longo do tempo (transiente).

Na figura 6 é observado os perfis de concentração de cada enantiômero ao longo das colunas quando o LMS atinge o estado estacionário, onde a boa representatividade da nova abordagem fica evidenciada devido a boa correlação com os dados experimentais e ainda pode ser observado que o para este caso o Front Velocity apresenta um ajuste ligeiramente melhor em relação as simulações de Santos et al. (2004).

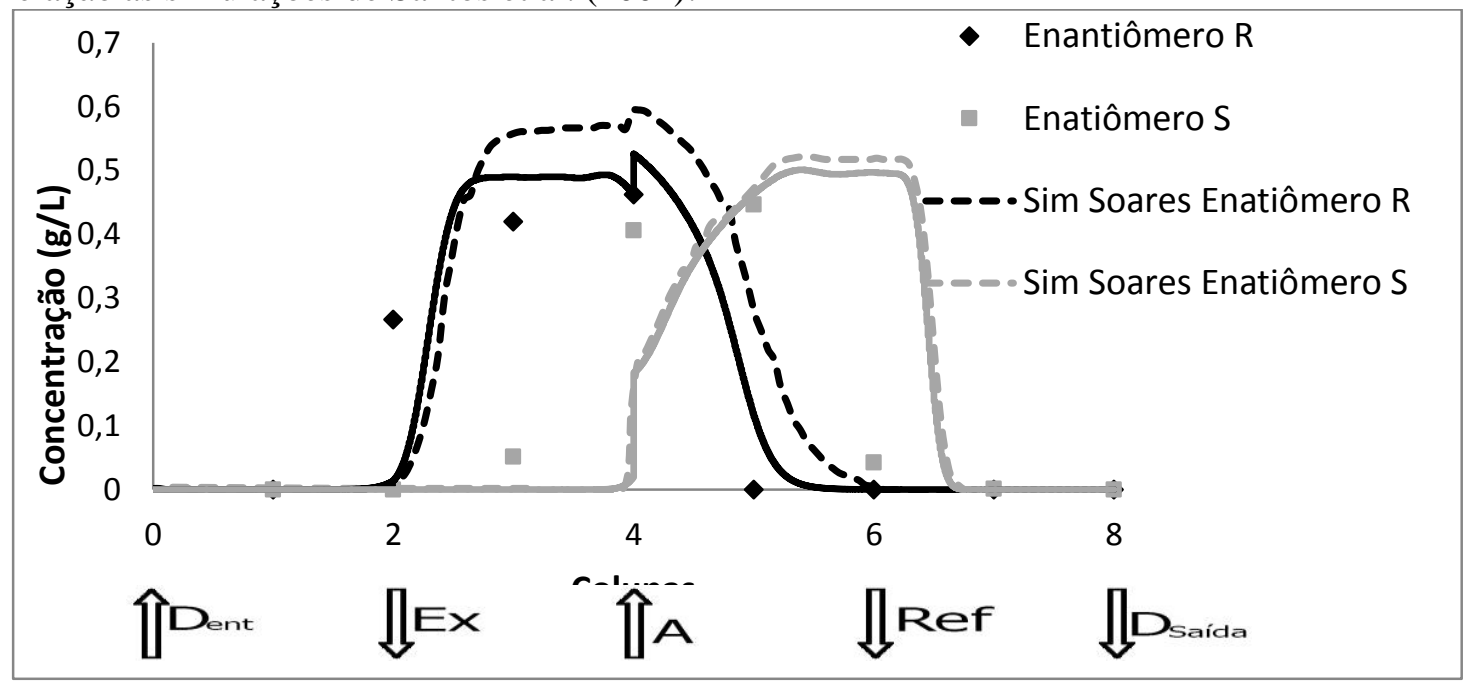

Figura 6:Perfil interno de concentração dos enantiômeros ao longo das colunas do LMS quando o equipamento atinge o estado estacionário.

\section{CONCLUSÕES}

Um conjunto de programas para a simulação contínua do equipamento LMS e também para a caracterização da coluna cromatográfica foi desenvolvida e utilizado sob a nova ideia proposta de modelar o experimento de pulso para determinar as constantes cinéticas de transferência de massa com equações de transporte de massa, em lugar de realizar experimentos de equilíbrio e combiná-los a algum tipo de isoterma de adsorção. Este procedimento reproduziu de forma satisfatória a separação dos enantiômeros do fármaco Cetamina em LMS. O algoritmo R2W mostrou-se efetivo na determinação dos parâmetros de adsorção e dessorção e foi capaz de 
ajustar até três parâmetros $\left(k_{a d s}, k_{\text {des }}, q_{m}\right)$ quando da aplicação do problema inverso à abordagem Front Velocity com cinética de Langmuir.

Os perfis de concentração das simulações mostraram-se coerentes com o processo LMS, e os perfis dos enantiômeros simulados se assemelharam com os dados experimentais, apresentando um comportamento levemente mais adequando em relação aos dados experimentais do que a própria modelagem clássica adotada por Santos.

Os resultado mostraram o potencial do Front velocity na predição de separações em LMS. O que torna esse modelo atrativo, é a simulação de todo o processo LMS em um curto intervalo de tempo devido ao baixo custo computacional, uma vez que as equações dos modelos são equações diferenciais ordinárias, ao contrário da abordagem clássica que se utiliza de equações diferenciais parciais as quais precisam um tratamento numérico especial. Também as novas abordagens propostas necessitam de um menor número de parâmetros em relação aos modelos clássicos, além da facilidade de implementação e análise e também a necessidade de conhecer poucos dados operacionais do problema real. Outro fato relevante é não ser necessária a realização de experimentos de equilíbrio para a caracterização da coluna cromatográfica.

\section{Referências}

[1] Broughton, D. B. (23 de Maio de 1961). EUA Patente No US002985589.

[2] Câmara, L. D., \& Silva Neto, A. J. (2008). Inverse Stochastic Characterization of Adsorption Systems by a Random Restricted Window (R2W) Method. International Conference on Engineering Optimization (ENGOPT). Rio de Janeiro - RJ.

[3] Gal, G., Hanak, L., Argyelan, J., Strbka, J., Szanya, T., Aranyi, A., \& Temesvari, K. (2005). Simulated Moving Bed (SMB) Separation of Pharmaceutical Enantiomers. Hungarian Journal of Industrial Chemistry, 33, pp. 23-30.

[4] Gomes, P. M., Figueirêdo, R. M., \& Queiroz, A. J. (2002). Caracterização e Isotermas de Adsorção de Umidade da Polpa de Acerola em Pó. Revista Brasileira de Produtos Agroindustriais, 4(2), pp. 157-165.

[5] Gonçalves, C. V. (2008). Separação do Racemato N-Boc-Rolipram pelo Processo Cromatografico de Leito Movel Simulado Utilizando Fase Estacionaria Quiral Tris-(3,5dimetilfenilcarbamato de celulose) Suportada em Silica. Campinas: UNICAMP: Tese de Doutorado.

[6] Hindmarsh, A. (1976). Preliminary Documentation of GEARIB - Solution of Implicit Systems of Ordinary Differential Equations with Banded Jacobian. Report UCID - 30130.

Madsen, N. K., \& Sincovec, R. F. (1979). PDCOL: General Collection Software for Partial Differential Equations. ACM Trans. Math. Software, 5, pp. 326 - 351.

[7] Santos, M. A. (2004). Separação dos Enantiômeros do Anestésico Cetamina por Cromatografia Contínua em Leito Móvel Simulado. Campinas - São Paulo: Tese de Doutorado, Unicamp.

[8] Santos, M. A., Veredas, V., Silva JR, I. J., Correia, C. R., Furlan, L. T., \& Santana, C. C. (2004). Simulated Moving-Bed Adsorption for Separation of Racemic Mixtures. Brazilian Journal of Chemical Engineering, 21(01), pp. 127-136.

[9] Zaijun, L., Xiulan, S., \& Junkang, L. (2011). Ionic Liquid as Novel Solvent for Extraction and Separation in Analytical Chemistry. Chemical Engineering Applications and Perspectives, pp. 154-180. 